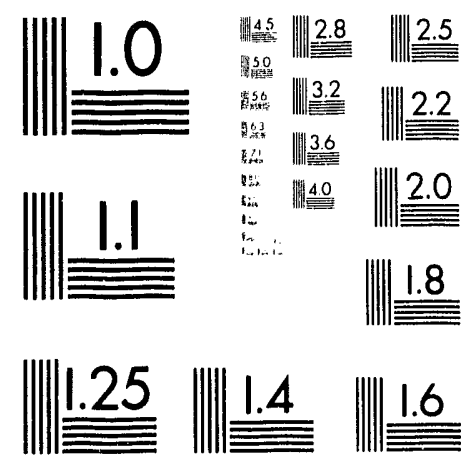



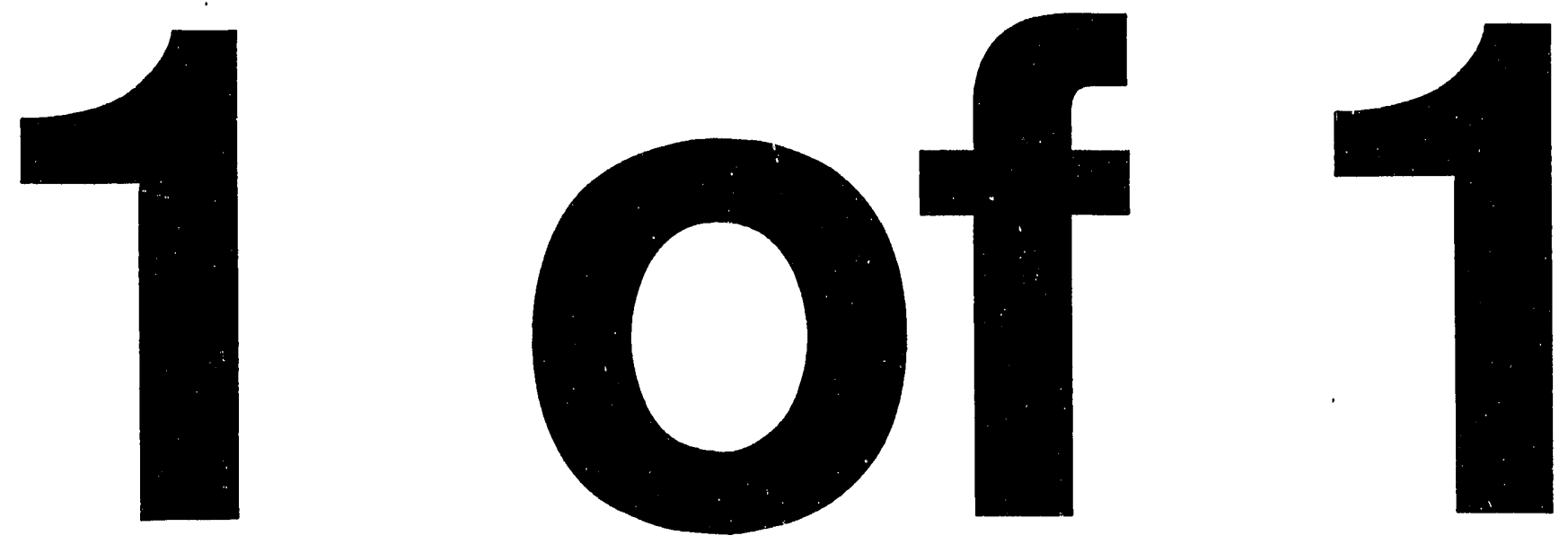


\title{
Conf $931018-16$
}

UCRL-JC-114268

\author{
PREPRINT
}

\section{Tokamak Physics Experiment Poloidal Field Design}

\author{
Richard H. Bulmer
}

This paper was prepared for submittal to the 15th IEEE/NPSS Symposium on Fusion Engineering Hyannis, MA

October 11-15, 1993

October 6, 1993

This is a preprint of a paper intended for publication in a journal or proceedings. Since changes may be made before publication, this preprint is made available with the understanding that it will not be cited or reproduced without the permission of the author.

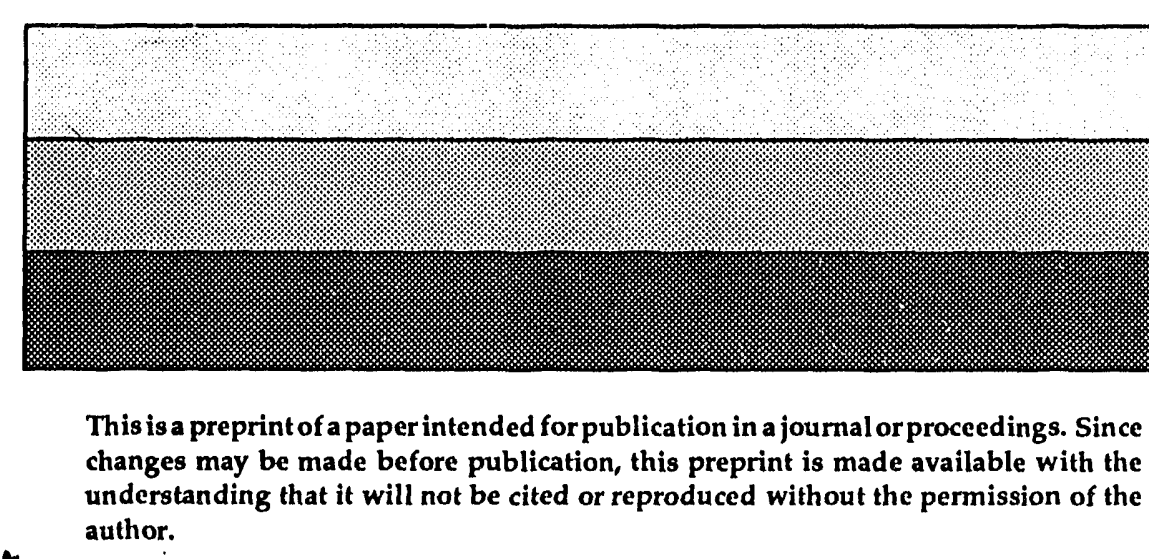




\section{DISCLAIMER}

This document was prepared as an account of work sponsored by an agency of the United States Government. Neither the United States Government nor the University of Callfornia nor any of their employees, makes any warranty, express or Implled, or assumes any legal liability or responsibliliy for the accuracy, completeness, or usefulnest of any Information, apparatus, product, or process disclosed, or represents that its use would not Infringe privately owned rights. Reference herein to any specific commerclal products, process, or service by trade name, trademark, manufacturer, or otherwise, does not necessarliy constitute or Imply its endorsement, recommendation, or favoring by the United States Government or the Unlversity of Californla. The vlews and opinions of authors expressed herein do not necesserlly state or reflect those of the United States Government or the Unlversity of California, and shall not be used for advertising or product endorsement purposes. 


\title{
Tokamak Physics Experiment Poloidal Field Design
}

\author{
Richard H. Bulmer \\ Lawrence Livermore National Laboratory \\ Livermore, CA 94550
}

\begin{abstract}
The Tokamak Physics Experiment (TPX) will have a poloidal field system capable of full inductive operation for approximately a 20 -s flattop and, with superconducting toroidal and poloidal field coils and non-inductive current drive, it will be capable of true steady-state operation. The poloidal field design is based on the ideal MHD equilibrium model as implemented in the TEQ code developed at LLNL. The PF coils are arranged in an up-down symmetric configuration, external to the TF coils. The TPX diverted plasma will have an aspect ratio of 4.5 and is highly shaped with a nominal elongation of 2 and triangularity of approximately 0.8 as measured at the separatrix. The tokamak design is based on a high-current $(q \psi=3)$ plasma scenario and a low current scenario. Each scenario has an operational fexibility requirement which is defined as a region of plasma pressure and inductivity $\left(\beta_{N}-\ell_{i}\right)$ space, where the plasma shape is constrained to keep the divertor configuration operational. Singlenull plasma configurations are feasible, even with the same divertor hardware, by operating the PF coils asymmetrically. Recently applied optimization techniques have improved the capability of the PF system without additional cost.
\end{abstract}

\section{INTRODUCTION}

The TPX poloidal field (PF) system design is developed from the free-boundary, axisymmetric, ideal MHD equilibrium model, as implemented in the TEQ [1] tokamak equilibrium and stability code. The code solves the GradShafranov equation within a constraint-equation solver enabling the arbitrary specification of a variety of plasma shapes and profile models. The primary purpose of these analyses is the determination of the external coil currents required to maintain prescribed equilibrium configurations. Surveys are performed over a wide range of parameter space to explore potentially interesting and accessible (from a PF coil limitation perspective) regions. Most of the equilibrium configurations of interest are X-point limited (i.e. poloidally diverted). Limited configurations are of interest primarily for start-up modeling and are not addressed here.
The nominal TPX plasma parameters are:

$\begin{array}{ll}\mathrm{B}_{\phi} & =4.00 \mathrm{~T} \\ \mathrm{R}_{o} & =2.25 \mathrm{~m} \\ a & =0.50 \mathrm{~m} \\ \mathrm{I}_{\mathrm{P}} & =2.00 \mathrm{MA} \\ \mathrm{x}_{x} & =2.00 \\ \delta_{x} & =0.80\end{array}$

where the toroidal field is measured at the major radius. Actual values of the elongation and triangularity, however, are explicitly defined by the intricacies of the divertor/limiter structures and therefore are a function of the plasma current and pressure profiles. These shape limitations will be described in detail in the following sections.

The PF system is designed for both up-down symmetric double-null (DN) and up-down asymmetric single-null (SN) configurations. Consequently, the PF coil configuration is geometrically up-down symmetric and SN equilibria are produced by asymmetric coil current distributions.

Two sets of double-null "design-basis" scenarios have been defined [2] which describe high-current (HC/DN) and low-current (LC/DN) fiducial discharges. The PF flexibility requirements for TPX are specified by ranges of normalized plasma pressure:

$$
\begin{aligned}
& \beta_{\mathrm{N}}=\frac{\beta_{\phi}}{\mathrm{I}_{\mathrm{p}} / a \mathrm{~B}} \phi_{\phi} \mathrm{mT} / \mathrm{MA} \\
& \text { and plasma normalized internal inductance: } \\
& \ell_{i}=\frac{2 V<\mathrm{B}_{\mathrm{P}}^{2}>}{\left(\mu_{o} \mathrm{I}_{\mathrm{P}}\right)^{2} R_{O}}
\end{aligned}
$$

Flexibility includes the capability to vary the elongation over a significant range. Surveys of $\kappa_{x}-\beta_{N}-\ell_{i}$ space are developed about nominal cases to map-out the required coil current distributions and subsequently the allowable operating regions for the plasma. Another important component of flexibility is the ability to produce strong single-null divertor configurations, where essentially all of the edge-loss power is deposited at the bottom divertorstructure.

Free-boundary equilibria of advanced plasma configurations are also evaluated to demonstrate the capability and flexibility of the PF system with specific, 
physics-relevant, operating scenarios. However, the highcurrent design-basis scenario (with full inductive currentdrive capability) sets the PF coil capability requirements.

\section{PF COIL CONFIGURATION}

The poloidal field coils are geometrically arranged in an up-down symmetric configuration as shown in Fig. 1. The coil set has the tlexibility to produce a broad range of DN configurations while maintaining the ability to create SN shapes by making the current distribution up-down asymmetric. There are two pairs of cutboard "ring coils", a pair of "divertor coils" and the central solenoid is divided into 4 pairs of modules. A 7-coil up-down symmetric connection is the nominal arrangement, and up to 14 individually controllable coil modules are available, although suitable $S N$ configurations can be produced with 10 independent PF circuits.

\section{INTERFACE CONSIDERATIONS}

This section describes the physical structures which define the spatial envelope in which all plasma shapes must be constrained to fit. The pertinent structures are: an outboard divertor-plate, an inboard divertor-plate, a beltlimiter and an inboard limiter.

The nominal scrape-off layer (SOL) allowance for double-null configurations is defined as the inboard and outboard envelopes between the separatrix surfaces mapped from the midplane. They are bounded by: (1) the flux surface which passes through a point $2-\mathrm{cm}$ outboard from the outer edge of the plasma $\left(\Delta R_{S O L}=2-\mathrm{cm}\right)$ and (2) the flux surface which passes through a point $5-\mathrm{cm}$ inboard from the inner edge of the plasma $\left(\Delta R_{S O L, i}=5-\mathrm{cm}\right)$.

\section{DIVERTED-PLASMA SHAPE CONSTRAINTS}

All of the equilibria described here maintain the inner and outer edge of the plasma at their nominal positions, corresponding to: $R_{o}=2.25-\mathrm{m}$ and $a=0.50-\mathrm{m}$, and the outboard strike-point coordinate is constrained. The strikepoint constraint effectively limits $\delta_{x}=0.8$, but the actual value of triangularity for any particular case is sensitive to profiles. The elongation, however, is controllable between upper and lower limits, as described below.

Excessive coil currents can also limit tis plasma clongation. Plasmas with high $\ell_{i}$ are increasingly hard to elongate, and plasmas with low $\ell_{i}$ may require prohibitive coil currents to reduce elongation. It is important to recognize that plasma profiles and plasma shape constraints must be mutually consistent -- otherwise an equilibrium may not necessarily exist. For example, very peaked current profiles $\left(\ell_{i}-2\right)$ are not compatible with highly elongated $\left(x_{x}-2\right)$ shapes.

The divertor plates and limiters restrict the maximum elongation $\left(x_{x, \max }\right)$ attainable for any given set of plasma parameters by one of three ways, as described below.
Fig. 2 depicts the shape constraints for a nominal plasma where the elongation is indirectly constrained by the outboard $\Delta R_{S O L}=2-\mathrm{cm}$ field line passing through the beltlimiter point. This constraint is the dominant one for most cases. There are two variations of the maximum elongation constraint, one where the plasma current is peaked (high $\ell_{i}$ ) and we use the $\Delta R_{S O L}=1 \mathrm{~cm}$ line and one where the plasma current is broad (low $\ell_{i}$ ) and the elongation is determined by the vertical extent of the inboard divertor structure.

\section{DESIGN-BASIS SCENARIOS}

The approach used to design the PF system for TPX is to define a small set of "design-basis" scenarios which are representative of idealized tokamak discharges. The designbasis scenarios represent two types of fiducial discharges, one at high plasma current and one at low plasma current. These two cases establish the reference equilibria from which flexibility requirements are defined.

The high-current scenario has $I_{\mathrm{P}}=2 \mathrm{MA}$ and the lowcurrent scenario has $I_{\mathrm{p}}=400 \mathrm{kA}$. Both design-basis scenarios are initiated at the same initial magnetization flux state $\left(\Psi_{I M}=9 \mathrm{~V}-\mathrm{s}\right)$. A set of four equilibria are generated for each of the two reference scenarios. These four equilibria correspond to the fiducial flux states: (1) start-offlattop (SOF), (2) start-of-beta (SOB), (3) end-of-beta (EOB) and (4) end-of-flattop (EOF). Further, states 1 and 4 and states 2 and 3 respectively only differ in external flux linkage (i.e. the plasma parameters are constrained to be the same) to cnable interpolation and/or extrapolation of coil currents at flux linkage values other than the values used to establish the equilibrium. This simplification allows the magnet designers to choose the flux linkage values for each operating scenario in an optimal fashion -.- balancing the capabilities of individual coils while providing sufficient flux-swing to reach full plasma current. The four fiducial equilibria are used to set up the nominal PF coil current waveforms for each design-basis set.

\section{PF SYSTEM FLEXIBILITY}

\section{Flexibility at High-Current}

A series of off-nominal equilibria (two dimensional surveys varying $\beta_{N}$ and $\ell_{i}$ ) have been generated to assess the operational flexibility of the PF system. The plasma current for these cases is set by:

$$
q_{9 S}=3 \text { except } / p \leq 2-\mathrm{MA}
$$

The desired pressure and current-profile flexibility range is:

$$
\begin{aligned}
& 0.0 \leq \beta_{N} \leq 5.0 \\
& 0.5 \leq l_{i} \leq 1.2
\end{aligned}
$$


at an elongation of $\boldsymbol{x}_{x, \max }$. The lower-left corner of this range is excluded, however, since vertical control is not feasible. Fig. 3 shows contour plots of certain PF coil currents as a function of $\beta_{\mathrm{N}}$ and $\ell_{i}$ for the maximum elongation configurations. These results were obtained at constant flux linkage. Note the magnitude of the current in PF1 increases with $\ell_{i}$ and is very sensitive to $\beta_{N}$ because the solenoid is trying to compensate for the changing plasma flux to hold the external flux linkage constant. The divertorcoil (PF5) is very sensitive at low $\ell_{i}$. The currents in the outer ring coils (PF6 and PF7) tend to trade-off as the plasma current profile varies. A similar set of flexibility requirements are defined at lower plasma current, $q_{95}=5$.

\section{Single-Null Flexibility}

The requirements for high-current single-null operation are patterned after the HC/DN design-basis. The following three additional constraints are applied to the plasma shape in single-null: (1) maintain the elevation of the magnetic axis at the DN position, (2) the "skew" of the poloidal flux surfaces (either at the axis or at the inboard edge) is eliminated and (3) the separation between active and inactive separatrices is constrained to be large enough to maintain true single-null divertor operation. We find we can achieve these single-null configurations, matched to the lower divertor structure, over the same $\ell_{i}$ range as in double null operation. Coil pairs PF4, PF5 and PF6 are frecd, making for 10 independent circuits.

\section{Elongation Flexibility}

The PF system will be designed with the plasma scenarios as described above, where in each case, the elongation is maximized within the spatial constraints. The lower limit on the plasma elongation (as set by the inboard divertor-plate structure) is approximately $\boldsymbol{x}_{x, \min }=1.6$. It is recognized that this lower limit may not be attainable for all current profiles, in particular, at low $\ell_{i}$ the PF coil current limits may prohibit operation at such low elongation.

\section{PF OPTIMIZATION}

Detailed assessment of the flexibility of the TPX PF coils uncovered some deficiencies in the design. The most scrious problem was due to the extraordinarily large current magnitude in PF7 with a peaked current profile (high $\ell_{i}$ ) and high $\beta_{N}$. Other deficiencies were also observed in PF5 and PF6, which were evident with broad plasma profiles (low $\ell_{i}$ ) and low pressure. Two criteria were used to assess coil capability, one was the fraction of critical current in the superconductor and the second was the magnitude of the heat transfer rate (convection coefficient) required to recover from a localized normal event. The TPX criteria for these two parameters are, respectively, $f_{c r i t} \leq 0.8$ and $h_{\text {recovery }} 51120 \mathrm{~W} / \mathrm{M}^{2} \mathrm{~K}$.
In an effort to improve the design of PF systems, Dennis Strickler at ORNL, has been developing a technique [3] of optimizing poloidal field coil positions and shapes. The technique uses the non-linear minimization of a cost function while matching (in a least-squares sense) the extemal field from a set of equilibria at points on the plasma boundary. The independent variables are the positions of the coils and the cross-sectional dimensions of the conductor packs. The coil positions determine how much current will be required to sustain a particular equilibrium, and the conductor pack size and aspect ratio are important parameters which determine the peak field at the conductor. The peak field, in turn, is a key parameter of the critical current in the superconductor, as well as affecting the magneto-resistance of the copper stabilizer which determines the amount of Joule heat generated in a normal event.

Partial results of the optimization technique applied to TPX are shown in Fig. 4, where the coil packs of the outboard ring coils have been elongated to reduce peak fields. With this minor modification of the conductor packs and altering of the plasma shape (less elongation and increased minor radius) at the high $\beta_{\mathrm{N}}$, high $\ell_{i}$ comer, we find a configuration where all the flexibility requirements are met with no additional cost on the magnet system.

\section{ACKNOWLEDGMENTS}

The author wishes to thank G. H. Neilson (of ORNL at PPPL) and the rest of the TPX physics team (particularly L. D. Pearlstein of LLNL) for guidance in this effort. The ongoing collaboration with D. J. Strickler at ORNL to optimize the PF coils has been very productive.

This work was performed under the auspices of the U.S. Department of Energy by Lawrence Livermore National Laboratory under contract number W-7405-ENG-48.

\section{REFERENCES}

[1] L. D. Pearlstein, Lawrence Livermore National Laboratory, Livermore CA, (private communication)

[2] TPX General Requirements Document, W. R. Reiersen, editor, PPPL, March 15, 1993.

[3] D. J. Strickler, Oak Ridge National Laboratory, Oak Ridge, TN (private communication) 


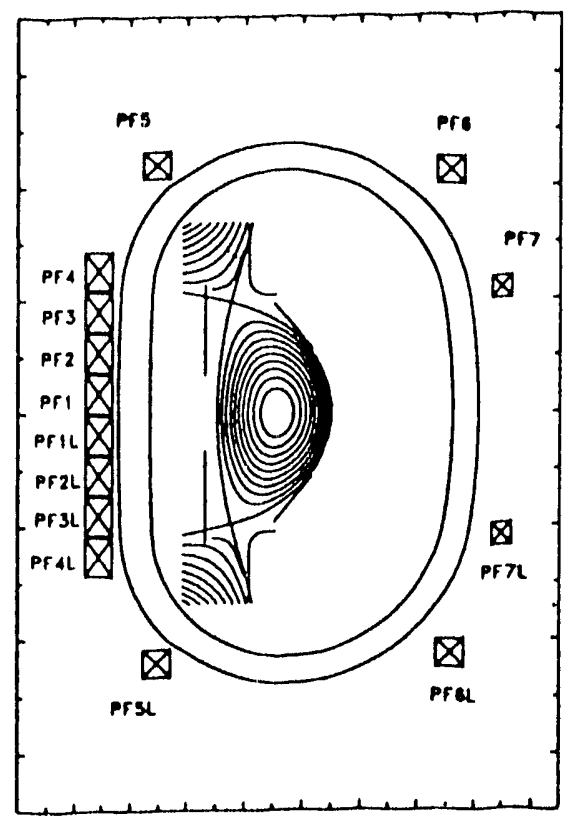

Figure 1.- TPX poloidal field coils, 7 pairs in an up/down symmetric arrangement, and reference equilibrium.
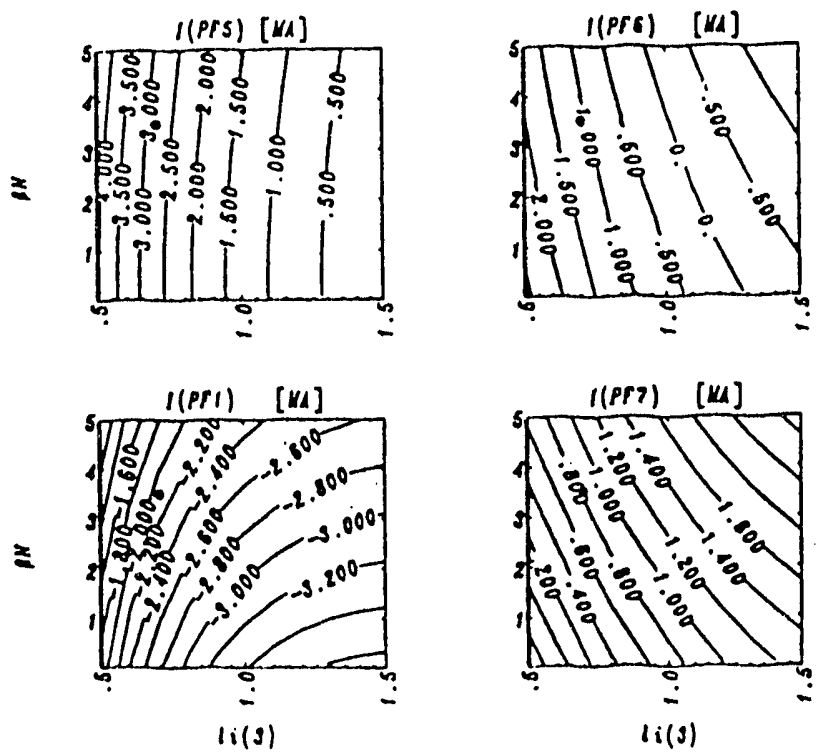

Figure 3 .. Variation in the current of some of the PF coils (PF1, PF5, PF6 AND PF7) over the desired nexibility range for one operating mode $(995=3)$ and one nux sule (FOB).

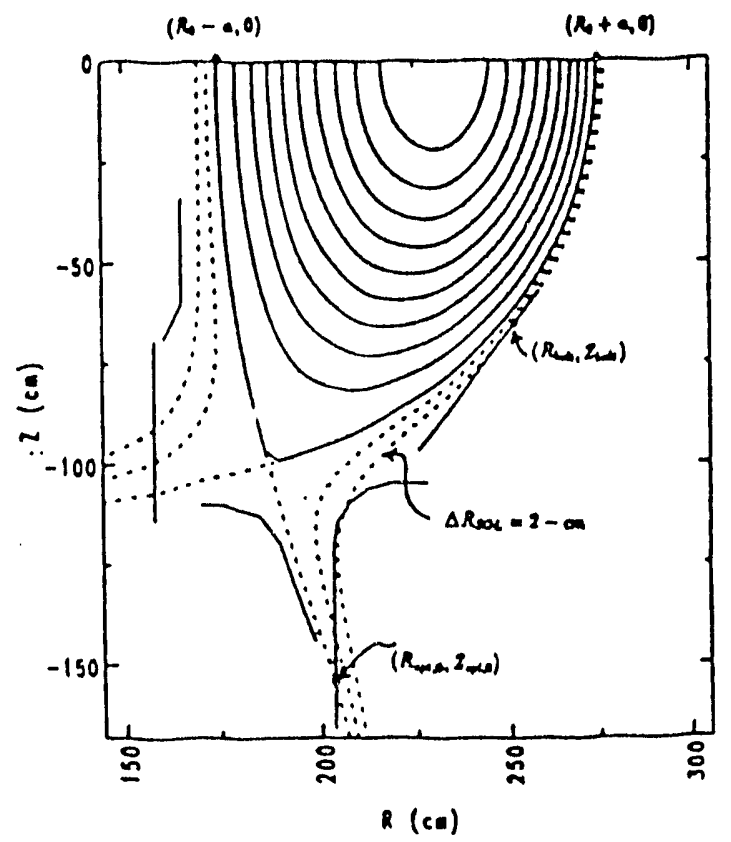

Figure 2 .. Four parameters: major radius, minor radius, outboard arikepoint and acoptance of the 2-cm scrape-off layer by the outboard belt-limiter def ine the four shape constrainis on the TPX plasma in most cases.

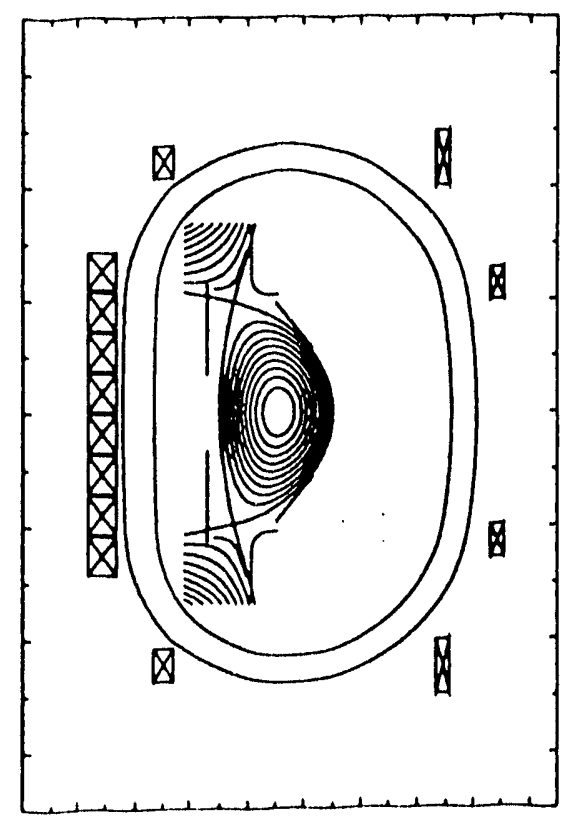

Figure 4 .. An opximized PF coil arrangement where elongating the conductor packs of the ring coils (PF5, PF6 and PF7) help reduce the peak field at the conductor. 

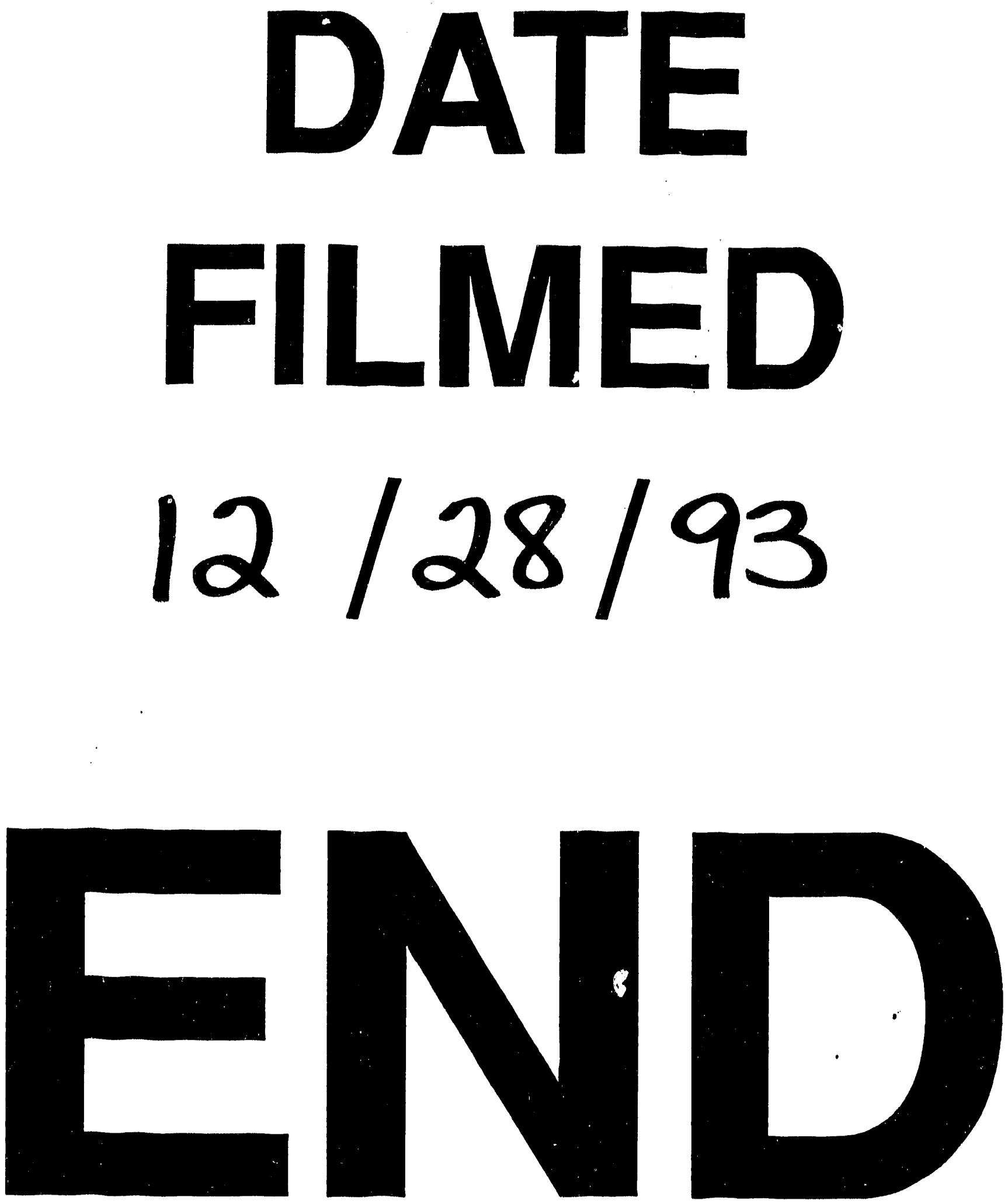
